\title{
The relationship of monocyte to high density lipoprotein-cholesterol ratio and complete blood count parameters with radiologic staging of knee osteoarthritis
}

\author{
Mustafa Fatih Yasar, Elif Yaksi \\ Department of Physical Medicine and Rehabilitation, Bolu Abant Izzet Baysal University, Faculty of Medicine, Bolu, \\ Turkey
}

\section{ABSTRACT}

\begin{abstract}
Aim: To evaluate the predictive ability of bioindicators derived from complete blood count (CBC) parameters and monocyte-high density lipoprotein-cholesterol ratio (MHR) in the diagnosis of radiological stage of knee osteoarthritis (OA) in this study.
\end{abstract}

Method: This cross-sectional retrospective study was carried out between November 2017 and June 2021, in our physical therapy and rehabilitation clinics. 65 patients knee x-rays and routine laboratory results were included in the study. Each knee x-rays were assigned a grade from 0 to 4 (Kellgren-Lawrence Classification System (KL). Patients were divided into two groups according to severity of the knee OA as follows. Group 1: Mild-moderate OA (KL Grade1-2), Group 2: Severe OA (KL Grade 3-4). Results: Independent $\mathrm{T}$ test and Mann Whitney $\mathrm{U}$ test were used to assess whether there was a difference in CBC parameters and their derivatives between two groups. Platelet lymphocyte ratio (PLR), red cell distribution width (RDW) to platelet ratio (RPR) and platelet (PLT) counts indicated statistically significant differences between the groups, $p$-values were $0.04,0.03$ and 0.04 respectively. There were no significant differences in terms of MHR score between the groups.

Conclusions: We could not find a relationship between MHR and radiological degree of knee osteoarthritis. However, there is a correlation between radiological stage of knee osteoarthritis and hemogram parameters like PLT and their derivatives such as PLR and RPR.

Key words: Knee, osteoarthritis, monocyte, high density lipoprotein, cholesterol, platelet, lymphocyte, red cell distribution.

$\triangle$ Dr. Mustafa Fatih Yasar

Department of Physical Medicine and Rehabilitation,

Bolu Abant Izzet Baysal University, Faculty of Medicine,

Bolu, Turkey

E-mail: mustafafy@hotmail.com

Received: 2021-09-28/Revisions: 2021-10-14

Accepted: 2021-10-28 / Published online: 2022-01-01

\section{Introduction}

Osteoarthritis (OA) is the most common cause of arthritis, characterized by progressive degeneration and destruction of joint cartilage. Genetic factors, old age, trauma, high body mass index (BMI), and deterioration of biomechanical properties are the main conditions that accelerate the process of osteoarthritis development. OA may remain silent for a long time or manifest slow progression. The disorder most commonly affects the knee joint symptomatically. Symptoms of OA include pain, joint dysfunction, and deformities. Knee $\mathrm{OA}$ is detected in approximately $80 \%$ of patients over 65 years old on plain radiographs; however, only one in three of these patients has 
symptoms [1]. Early diagnosis of the disease and estimation of the clinical course and prognosis is difficult due to long asymptomatic periods. Recent studies have focused on discovering reliable biomarkers in the diagnosis and progression of OA [2,3]. Until recently, OA was accepted as a degenerative disease caused by joint abrasion and degeneration of periarticular soft tissue due to aging and increased mechanical load. Novel studies have proven that $\mathrm{OA}$ is associated with low-grade chronic inflammation [4-7]. Low-grade inflammation contributes to the pathogenesis of $\mathrm{OA}$ by inducing the production of proinflammatory cytokines. This process involves mononuclear cell (monocytes, macrophages, and activated $\mathrm{T}$ and $\mathrm{B}$ lymphocytes) infiltration in joint space and the release of proinflammatory mediators, such as IL-1 $\beta$ and TNF. These mediators stimulate the production of matrix-metalloproteinases (MMPs), prostaglandin E2 (PGE2), nitric oxide (NO), and some other cytokines (IL-6, IL-8, IL-15, IL-17, IL-21). IL-1, IL-6, and TNF, which are key cytokines involved in the inflammatory process, may also lead to platelet activation. Platelets release inflammatory mediators and growth factors during hemostasis, inflammation, and tissue repair [8,9]. Monocytes and neutrophils contribute to inflammatory process steps and oxidative stress by releasing pro-inflammatory and prooxidative cytokines [10]. In recent years, various hematologic parameters, including mean platelet volume (MPV), neutrophillymphocyte ratio (NLR), platelet-lymphocyte ratio (PLR), and monocyte-high density lipoprotein-cholesterol ratio (MHR), have been evaluated as indicators of inflammation $[11,12]$. Previous studies have shown that high density lipoprotein-cholesterol (HDL-C) has anti-inflammatory, anti-oxidant effects and protects endothelial cells $[13,14]$. Parameters such as MHR and NLR and red cell distribution width-platelet ratio (RPR) can be utilized by routine laboratory tests. These ratios can be computed by using complete blood count (CBC) results and biochemical profiles easily and inexpensively.

These parameters have been evaluated as indicators of inflammation and oxidative stress for systemic inflammatory diseases and cardiovascular disorders [15].

NLR has been introduced as a marker of inflammation in irritable bowel syndrome, ulcerative colitis, thyroiditis, and type 2 diabetes mellitus [16-19]. PLR has also been increased in various inflammatory conditions, including malignancy [20].

There is a paucity of research about the clinical and radiological progression of OA. Therefore, we aimed to assess the predictive ability of bioindicators derived from $\mathrm{CBC}$ parameters and the MHR ratio in the diagnosis of the radiological stage of knee OA in this study.

\section{Materials and methods}

\section{Ethics statement}

This cross-sectional retrospective study was carried out between November 2017 and June 2021, in our physical therapy and rehabilitation clinics. The study protocol was approved by our Institutional Review Board (Ethics Committee Approval Date and Number: 08.06/2021-148) and conducted in accordance with the Declaration of Helsinki.

\section{Study design and population}

\section{Inclusion criteria}

Patients with primary knee OA according to the clinical criteria of the American College of Rheumatology (ACR) and between the ages of 40-80 were included in the study. If OA wasn't unilateral, only the patients with an equal degree of OA on both knees were included. 
All diagnostic decisions were made by the same physician. 65 patients with standing, weight bearing anteroposterior and lateral knee $\mathrm{x}$-rays and routine laboratory results were included in the study. Each knee x-rays was assigned a grade from 0 to 4 (Kellgren-Lawrence Classification System (KL)). Demographic information and body mass index of the patients with knee OA were recorded.

Patients were divided into two groups according to severity of the knee OA as follows. Group 1: Mild-moderate OA (KL Grade1-2)

Group 2: Severe OA (KL Grade 3-4)

\section{Kellgren-Lawrence classification system}

The Kellgren-Lawrence system is applied specifically to classify the severity of knee OA using five grades and originally described using AP knee radiographs. This classification system was accepted by WHO in 1961. Below is the original description.

Grade 0: No radiographic features of OA are present

Grade 1: Doubtful joint space narrowing (JSN) and possible osteophytic lipping

Grade 2: Definite osteophytes and possible JSN on anteroposterior weight-bearing radiograph

Grade 3: Multiple osteophytes, definite JSN, sclerosis, possible bony deformity

Grade 4: Large osteophytes, marked JSN, severe sclerosis and definite bony deformity

\section{Exclusion criteria}

Participants with hematologic disorders which may affect blood parameters, patients with diagnosis of diabetes mellitus, acute decompensation and organ failure, inflammatory diseases, rheumatic diseases, acute or chronic infection, cardiovascular diseases, secondary osteoarthritis, oncologic diseases, patients who are receiving intraarticular steroid or hyaluronic acid treatment, patients with history of severe knee trauma or operation last 6 months, patients during the pregnancy and lactation period were excluded from participation.

\section{Laboratory analysis}

While low density lipoprotein cholesterol [LDL-C] (mg/dL), and high density lipoprotein cholesterol [HDL-C] (mg/dL) levels were obtained from biochemical analysis (Architect CI4100, Chicago, USA), white blood cell (WBC) $(\mathrm{K} / \mathrm{uL})$, neutrophil $(\mathrm{K} / \mathrm{uL})$, lymphocyte $(\mathrm{K} / \mathrm{uL})$, monocyte $(\mathrm{K} / \mathrm{uL})$, hemoglobin $(\mathrm{Hb})$ (g/dL), red blood cell distribution width (RDW), platelet (K/uL) (PLT) and mean platelet volume (MPV) (fl), platelet distribution width (PDW) values were acquired from CBC analysis using an XT1800I Kobe, JAPAN hematology analyzer. On the basis of these test results, NLR, PLR, LMR and MHR were calculated as dividing by different parameters. All assays were performed according to the manufacturer's' instructions.

\section{Statistical analyses}

The data obtained in the study were analyzed on SPSS statistical package (IBM SPSS Statistics for Windows, Version 26.0. Armonk, NY: IBM Corp). Mean values, standard deviation values were calculated for all parameters. Kolmogorov-Smirnov test is used to assess normality of the variables. Independent $\mathrm{T}$ tests were used to compare the means between the groups for normally distributed data. Chisquare test is performed to understand the relationship of categorical variables. A p-value less than 0.05 is considered statistically significant.

\section{Results}

\section{Demographic characteristics}

Overall, 65 patients between the ages of 40-80, including 35 with mild-moderate $\mathrm{OA}$ and 30 with severe OA, were assigned to our study. Patients ranged in age from 44 to 80 , with a mean age of $65.7 \pm 9.4$. Most participants were 
Table 1: Demographic information.

\begin{tabular}{|c|c|c|c|c|}
\hline Parameters & $\begin{array}{c}\text { Group 1 } \\
\text { (Mild-Moderate Osteoarthritis) }\end{array}$ & $\begin{array}{c}\text { Group 2 } \\
\text { (Severe Osteoarthritis) }\end{array}$ & Total & $p$ \\
\hline Mge & & & & \\
\hline Min-Max & $62.5 \pm 8.6$ & $69.4 \pm 9.1$ & $65.7 \pm 9.4$ & 0.79 \\
\hline Gender & $46-77$ & $44-80$ & $44-80$ & \\
\hline Female (n, \%) & $21(60 \%)$ & $21(70 \%)$ & $42(64 \%)$ & 0.10 \\
\hline Male (n, \%) & $14(40 \%)$ & $9(30 \%)$ & $23(35 \%)$ & \\
\hline Admission status & $11(31 \%)$ & $7(23 \%)$ & $18(28 \%)$ & \\
\hline In-patient (n, \%) & $24(67 \%)$ & $23(77 \%)$ & $47(72 \%)$ & \\
\hline Out-patient (n, \%) & & & & \\
\hline
\end{tabular}

SD: standard deviation, Min: minimum, Max: maximum

female (64\%). Demographic characteristics were similar between the two groups $(\mathrm{P}>0.05)$. Most patients (72\%) were admitted to our outpatient clinics, and the remaining patients were hospitalized. Detailed information about the demographics of the participants is available in Table 1.

\section{Prediction of OA grade according to hemogram parameters and MHR}

Independent $\mathrm{T}$ test and Mann Whitney $\mathrm{U}$ test were used to assess whether there was a difference in CBC parameters and their derivatives between the two groups. PLR, RPR ratios, and PLT counts indicated statistically significant differences between the groups; $\mathrm{p}$ values were $0.04,0.03$, and 0.04, respectively. The area under the curve for PLR and RPR was computed as 0.644 and 0.374 , respectively (Figure 1). There were no significant differences in terms of MHR score between the groups; p-values were 0.07. The rest of the factors did not show a statistically significant difference between the groups (Table 2).

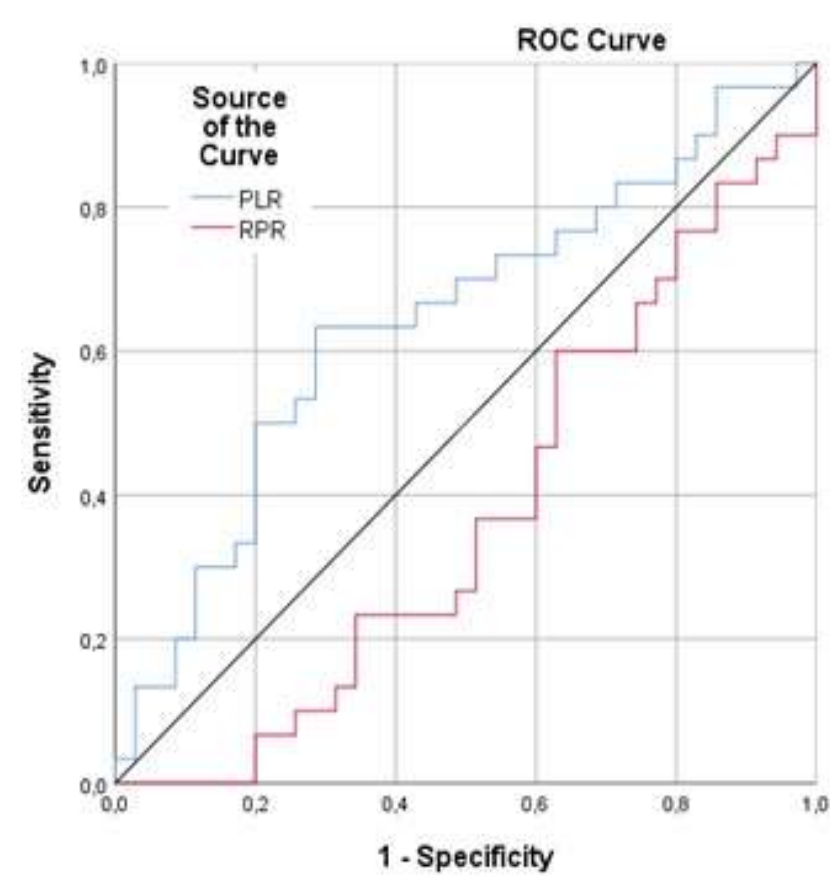

Figure 1. ROC curve analysis of the test (PLR curve:blue line, RPR curve:red line, reference: black line).

\section{Discussion}

The major findings of our study, in which we evaluated the predictive performance of bioindicators like blood parameters and MHR in the diagnosis and staging of knee osteoarthritis, were as follows: 
Table 2. Comparison of the laboratory test results of the groups.

\begin{tabular}{|l|c|c|c|}
\hline Parameters & $\begin{array}{c}\text { Group 1 } \\
(\mathrm{n}=35)\end{array}$ & $\begin{array}{c}\text { Group 2 } \\
(\mathrm{n}=30)\end{array}$ & $\boldsymbol{P}$ \\
\hline WBC & $6.70 \pm 1,5$ & $7.04 \pm 1,8$ & 0.75 \\
\hline Neu & $3.75 \pm 1,3$ & $4.18 \pm 1,5$ & 0.47 \\
\hline Lym & $2.13 \pm 0,5$ & $2.10 \pm 0,7$ & 0.87 \\
\hline Mono & $0.46 \pm 0,1$ & $0.52 \pm 0,2$ & 0.17 \\
\hline HB & $13.4 \pm 1,5$ & $13.2 \pm 1,3$ & 0.37 \\
\hline RDW & $14.58 \pm 1,3$ & $14.65 \pm 1,2$ & 0.85 \\
\hline PLT & $221.4 \pm 56,6$ & $252.0 \pm 53,2$ & $\mathbf{0 . 0 4}$ \\
\hline HDL & $50.6 \pm 11,2$ & $47.5 \pm 10,3$ & 0.34 \\
\hline LDL & $129.3 \pm 34,9$ & $119.4 \pm 28,9$ & 0.27 \\
\hline NLR & $1.82 \pm 0,7$ & $2.11 \pm 0,9$ & 0.25 \\
\hline PLR & $110.50 \pm 40,2$ & $132.6 \pm 47,8$ & $\mathbf{0 . 0 4}$ \\
\hline RPR & $0.07 \pm 0.022$ & $0.06 \pm 0.01$ & $\mathbf{0 . 0 3}$ \\
\hline MHR & $0.009 \pm 0.003$ & $0.011 \pm 0.005$ & 0.07 \\
\hline MLR & $0.22 \pm 0.08$ & $0.26 \pm 0.10$ & 0.18 \\
\hline
\end{tabular}

Values: Mean $\pm S D$. Group 1: Grade 1, 2 osteoarthritis. Group 2: Grade 3, 4 osteoarthritis. SD: Standard deviation, WBC: White blood cell. Lym: Lymphocyte. Mono: Monocyte. Neu: Neutrophil. HB: Hemoglobin. RDW: Red cell distribution width. PLT: Platelet. HDL: High density lipoprotein. LDL: Low density lipoprotein. NLR: Neutrophil/Lymphocyte ratio. PLR: Platelet/Lymphocyte ratio. RPR: RDW/Platelet ratio. MHR: Monocyte/HDL ratio. MLR: Monocyte/Lymphocyte ratio.

1. A significant difference was detected in terms of PLT, PLR and RPR values.

2. There were no significant differences in terms of MPV, MHR, NLR score between the groups.

3. There were no significant differences in terms of HDL and LDL levels between the groups.

Previous studies showed that hemogram parameters and their derivatives, such as MPV, NLR, and PLR, could indicate systemic inflammation in various etiologies [21-23].
MPV is one test run during the CBC; NLR and PLR can be easily calculated by dividing neutrophil or platelet count by lymphocyte count. In the literature, there seem to be limited studies on the relationship between OA and PLT parameters. PLT indices may reflect subclinical inflammation in $\mathrm{OA}$ and disease activation. What is known about the PLT activation process is not only that the number of PLT increases, but also that to reach a larger surface area, their morphology changes from discoid to spherical patterns [24]. Also, larger 
platelets are likely to be more active at releasing pro-inflammatory and thrombotic mediators, and increased demand is seen during acute phase response [25].

Several studies have been conducted on the diagnostic and prognostic ability of $\mathrm{CBC}$ parameters and their derivatives, such as MPV, RDW, and RPR, during infectious and inflammatory disorders [26,27]. It has been proposed that there is a link between high MPV and RDW values and mortality or morbidity due to vascular events affecting the heart and kidneys, resulting in infectious disease [28-30]. Although many attempts have been made to assess the relationship between MPV and systemic inflammatory disorders, the results are contradictory [31,32]. It has been reported that MPV values in patients with active rheumatoid arthritis (RA) or ankylosing spondylitis (AS) are lower than in healthy controls. However, it is recommended to be careful when interpreting the MPV values, because this ratio is not consistently related to disease activity indexes, and tests may be influenced by technical settings [33,34]. Balbaoglu et al. compared patients with a diagnosis of OA, patients with synovitis related to $\mathrm{OA}$, and a control group; there was no statistically significant difference detected between all groups. On the contrary, MPV values in patients with synovitis related to OA were lower than in the other groups [31].

Koca et al. reported that MPV value was increased in patients with severe osteoarthritis [32]. Despite the Koca et al. findings, a recent study conducted by Atar et al. found that NLR, PLR, and MPV values were similar between 92 knee OA patients and 52 healthy controls [32,35]. Our results share a similarity with Atar et al.'s findings; no significant correlation was observed between MPV values and OA grades. PLR, which is calculated as absolute platelet count dividing by absolute lymphocyte count, is another principal factor with an influence on systemic inflammatory response pathogenesis [36].

In contradiction with earlier findings, we found a relationship between PLR ratio, knee osteoarthritis degree, PLT, and RPR levels in patients with advanced knee osteoarthritis [35]. NLR, as a marker of systemic inflammation, may reflect the neutrophil \& lymphocyte balance and immune system status of the patient [37].

Tasoglu et al. compared NLR ratios of 176 patients with $\mathrm{OA}$ according to the KellgrenLawrence classification and found higher NLR values in patients with severe OA (grade 4). They suggest that the NLR ratio can be used as a predictor tool to estimate the radiological grade of knee OA [3]. Büyükavc1 et al. proposed using blood platelet distribution width (PDW) and NLR to predict the severity of knee OA in clinical practice [38]. Atar et al. did not find a significant correlation between NLR ratio and radiological severity of knee osteoarthritis [3,35-38]. Similar to Atar et al.'s study, we did not find a relationship between the radiological stage of knee OA and NLR [35]. Contradictory results may be explained by local inflammation without systemic response. Future studies with larger populations should concentrate on this topic.

To the best of our knowledge, this is the first study in the literature evaluating the association between the MHR ratio, which is a novel bioindicator recently used as a marker of inflammation, and knee osteoarthritis.

The mononuclear phagocyte system plays a fundamental role in the inflammatory response during the development and progression of atherosclerosis. In the premature steps of the process, circulating monocytes migrate to the subendothelial matrix of the arterial wall, mature into macrophages, and internalize 
oxidized LDLs and other lipids. Subsequently, these cells transform to foam cells, which release immunoregulatory cytokines, trigger inflammation, and invite T-lymphocytes, platelets, and further monocytes [39]. However, HDL-C disrupts monocyte functions, interferes with the conversion of monocytes to macrophages, and removes cholesterol from them, which reduces the inflammatory response [40]. Therefore, it is appropriate to utilize the combination of both parameters (MHR) in a single index as a pro-inflammatory marker. Previous studies recommended using MHR as a predictive marker of cardiovascular disease due to its ability to demonstrate systemic inflammation [41]. Demirbaş et al. showed a significant decrease in MHR, monocyte to lymphocyte ratio (MLR), and NLR levels after colchicine treatment [42].

Sirin et al. revealed that the Psoriasis Area and Severity Index score (PASI) was associated with C-reactive protein, serum amyloid $\mathrm{A}$, and MHR in their study of 60 psoriasis vulgaris patients and 50 healthy controls. Our findings do not support previous studies, and we did not find a relationship between MHR and radiological grade of knee osteoarthritis [43]. The retrospective design of the study and the limited number of participants may be the reason for this concordance.

Recently, it has been thought that osteoarthritis may be a metabolic disease and lipid metabolism defects may be one of the underlying mechanisms $[44,45]$. Schwager et al. investigated the effects of serum cholesterol, low-density lipoprotein, and high density lipoproteins on the risk of knee osteoarthritis. As a result, they reported that they did not find a significant relationship between HDL, LDL and total cholesterol levels, and cartilage loss or worsening pain. [46]. Irshad et al. reported that serum cholesterol and triglyceride levels were associated with osteoarthritis, but there was no relationship between serum HDL and LDL levels and osteoarthritis [47]. Like these studies, no significant correlation was found with HDL and LDL levels in our study.

Advanced age is a non-modifiable risk factor associated with OA. It is predictable that radiologically advanced OA patients will be older than those with low-grade OA; hence, age-dependent alterations in patients' laboratory results show concomitance with radiologic advanced stages.

We know our study may have a few limitations. The first is that the retrospective design of the study may have influenced the assessment of the clinical presence of inflammation. Another limitation is that there was no control group to compare the results with patients. The small sample size is also a limitation of this study.

\section{Conclusions}

The evidence from our study suggests that there is a correlation between the radiological stage of knee osteoarthritis and hemogram parameters like PLT and their derivatives such as PLR and RPR. However, we could not find a relationship between NLR, MHR, MPV, and radiological degree of knee osteoarthritis. Further work needs to be carried out in a larger patient group with more clinical evaluation parameters.

Funding: The author(s) received no financial support for the research, authorship, and/or publication of this article.

Conflict of Interest: The authors declare that they have no conflict of interest.

Ethical statement: This study protocol was approved by Bolu Abant Izzet Baysal University Clinic Review Board (Date and Number: 08.06/2021-148), and conducted in accordance with the Declaration of Helsinki. 


\section{Open Access Statement}

This is an open access journal which means that all content is freely available without charge to the user or his/her institution under the terms of the Creative Commons Attribution NonCommercial License (https://creativecommons.org/licenses/by/4.0/). Users are allowed to read, download, copy, distribute, print, search, or link to the full texts of the articles, without asking prior permission from the publisher or the author.

Copyright (c) 2021: Author (s).

\section{References}

[1]Felson DT, Naimark A, Anderson J, et al. The prevalence of knee osteoarthritis in the elderly. The Framingham Osteoarthritis Study. Arthritis Rheum. 1987; 30(8):914-18. [2]Zhai G, Randell EW, Rahman P. Metabolomics of osteoarthritis: emerging novel markers and their potential clinical utility. Rheumatology (Oxford). 2018; 57 (12): 2087-95.

[3]Tasoglu O, Boluk H, Sahin Onat S, et al. Is blood neutrophil-lymphocyte ratio an independent predictor of knee osteoarthritis severity? Clin Rheumatol. 2016; 35(6): 1579-83.

[4]Sellam J, Berenbaum F. The role of synovitis in pathophysiology and clinical symptoms of osteoarthritis. Nat Rev Rheumatol. 2010; 6 (11):625-35.

[5]Goldring MB, Otero M. Inflammation in osteoarthritis. Curr Opin Rheumatol. 2011; 23 (5): 471-78.

[6]Benito MJ, Veale DJ, FitzGerald O, et al. Synovial tissue inflammation in early and late osteoarthritis. Ann Rheum Dis. 2005;64 (9): 1263-67.

[7]Robinson WH, Lepus CM, Wang Q, et al. Low-grade inflammation as a key mediator of the pathogenesis of osteoarthritis. Nat Rev Rheumatol. 2016; 12(10): 580-92.

[8]Dan K, Gomi S, Inokuchi K, et al. Effects of interleukin-1 and tumor necrosis factor on megakaryocytopoiesis: mechanism of reactive thrombocytosis. Acta Haematol. 1995; 93(2-4): 67-72.

[9]Unsal E, Aksaray S, Koksal D, et al. Potential role of interleukin 6 in reactive thrombocytosis and acute phase response in pulmonary tuberculosis. Postgrad Med J. 2005; 81 (959): 604-7.

[10] Ancuta P, Wang J, Gabuzda D. CD16+ monocytes produce IL-6, CCL2, and matrix metalloproteinase-9 upon interaction with CX3CL1-expressing endothelial cells. J Leukoc Biol. 2006; 80(5): 1156-64.

[11]36th International Symposium on Intensive Care and Emergency Medicine: Brussels, Belgium. 15-18 March 2016 (2016). Crit Care 20 (Suppl 2):94.

[12] Imtiaz F, Shafique K, Mirza SS, et al. Neutrophil lymphocyte ratio as a measure of systemic inflammation in prevalent chronic diseases in Asian population. Int Arch Med. 2012; 5(1): 2.

[13]Hessler JR, Robertson AL Jr, Chisolm GM, 3rd. LDL-induced cytotoxicity and its inhibition by HDL in human vascular smooth muscle and endothelial cells in culture. Atherosclerosis. 1979; 32(3): 21329.

[14]Parthasarathy S, Barnett J, Fong LG. Highdensity lipoprotein inhibits the oxidative modification of low-density lipoprotein. Biochim Biophys Acta. 1990; 1044 (2): 27583.

[15]Canpolat U, Cetin EH, Cetin S, et al. Association of Monocyte-to-HDL Cholesterol Ratio with Slow Coronary Flow is Linked to Systemic Inflammation. Clin Appl Thromb Hemost. 2016; 22(5): 476-82. 
[16] Aktas G, Duman TT, Atak BM, et al. Irritable bowel syndrome is associated with novel inflammatory markers derived from hemogram parameters. Fam Med Prim Care Rev. 2020; 22(2): 107-110.

[17]Posul E, Yilmaz B, Aktas G, et al. Does neutrophil-to-lymphocyte ratio predict active ulcerative colitis? Wien Klin Wochenschr. 2015;127(7-8):262-65.

[18]Aktas G, Sit M, Dikbas O, et al. Elevated neutrophil-to-lymphocyte ratio in the diagnosis of Hashimoto's thyroiditis. Rev Assoc Med Bras (1992). 2017;63(12):106568.

[19]Bilgin S, Aktas G, Zahid Kocak M, et al. Association between novel inflammatory markers derived from hemogram indices and metabolic parameters in type 2 diabetic men. Aging Male. 2020;23(5):923-27.

[20] Atak Tel BM, Kahveci G, Bilgin S, et al. Platelet to lymphocyte ratio in differentiation of benign and malignant thyroid nodules. Exp Biomed Res 2021; 4(2):148-53.

[21] Kilincalp S, Coban S, Akinci $H$, et al. Neutrophil/lymphocyte ratio, platelet/lymphocyte ratio, and mean platelet volume as potential biomarkers for early detection and monitoring of colorectal adenocarcinoma. Eur J Cancer Prev. 2015; 24(4): 328-33.

[22] Kuyumcu ME, Yesil Y, Ozturk ZA, et al. The evaluation of neutrophil-lymphocyte ratio in Alzheimer's disease. Dement Geriatr Cogn Disord. 2012; 34 (2): 69-74.

[23] Boyraz I, Koc B, Boyaci A, et al. Ratio of neutrophil/lymphocyte and platelet/lymphocyte in patient with ankylosing spondylitis that are treating with anti-TNF. Int J Clin Exp Med. 2014; 7(9): 2912-15.
[24] Jagroop IA, Clatworthy I, Lewin J, et al. Shape change in human platelets: measurement with a channelyzer and visualisation by electron microscopy. Platelets. 2000; 11(1): 28-32.

[25] Schmitt-Sody M, Metz P, Gottschalk O, et al. Platelet P-selectin is significantly involved in leukocyte-endothelial cell interaction in murine antigen-induced arthritis. Platelets. 2007; 18 (5): 365-72.

[26]Zareifar S, Farahmand Far MR, Golfeshan F, et al. Changes in platelet count and mean platelet volume during infectious and inflammatory disease and their correlation with ESR and CRP. J Clin Lab Anal. 2014; 28(3): 245-48.

[27] Cetinkaya E, Senol K, Saylam B, et al. Red cell distribution width to platelet ratio: new and promising prognostic marker in acute pancreatitis. World J Gastroenterol. 2014; 20 (39):14450-54.

[28] Patel KV, Semba RD, Ferrucci L, et al. Red cell distribution width and mortality in older adults: a meta-analysis. J Gerontol A Biol Sci Med Sci. 2010; 65(3): 258-65.

[29]Zampieri FG, Ranzani OT, Sabatoski V, et al. An increase in mean platelet volume after admission is associated with higher mortality in critically ill patients. Ann Intensive Care. 2014; 4:20.

[30] Atakan-Erkal F, Sepin-Özen N, Emek M, et al. Increased mean platelet volume, red blood cell distribution width and platelet / red blood cell distribution width in patients with Hepatitis B. Turk Hij Den Biyol Derg. 2019; 76(1): 53-58.

[31] Balbaloglu O, Korkmaz M, Yolcu S, et al. Evaluation of mean platelet volume (MPV) levels in patients with synovitis associated with knee osteoarthritis. Platelets. 2014; 25(2): 81-85. 
[32] Koca TT, Baykara M, Koçyiğit BF. Relation of complete blood count parameters and derivatives with radiologic staging of knee osteoarthritis. Cukurova Med J. 2019; 44 (4):1364-70.

[33] Kisacik B, Tufan A, Kalyoncu U, et al. Mean platelet volume (MPV) as an inflammatory marker in ankylosing spondylitis and rheumatoid arthritis. Joint Bone Spine. 2008; 75 (3): 291-4.

[34] Sahin A, Yetisgin A, Sahin M, et al. Can Mean Platelet Volume $\mathrm{Be}$ a Surrogate Marker of Inflammation in Rheumatic Diseases? West Indian Med J. 2015; 65(1): 165-69.

[35] Atar E, Aşkın A. Diz osteoartrit hastalarında nötrofil/lenfosit oran1, trombosit/lenfosit oranı ve ortalama trombosit hacminin değerlendirilmesi. Cukurova Med J. 2017; 42 (2): 329-36.

[36]Liu H, Wu Y, Wang Z, et al. Pretreatment platelet-to-lymphocyte ratio (PLR) as a predictor of response to first-line platinumbased chemotherapy and prognosis for patients with non-small cell lung cancer. J Thorac Dis. 2013; 5(6): 783-89.

[37]Lissoni P, Brivio F, Fumagalli L, et al. Efficacy of cancer chemotherapy in relation to the pretreatment number of lymphocytes in patients with metastatic solid tumors. Int J Biol Markers. 2004;19 (2): 135-40.

[38] Buyukavci R, Akturk S, Sag S. Comparison of blood platelet distribution width and neutrophil-lymphocyte ratio in patients with different grades of knee osteoarthritis. J Back Musculoskelet Rehabil. 2018; 31(6): 1035-39.

[39] Ganjali S, Gotto AM, Jr., Ruscica M, et al. Monocyte-to-HDL-cholesterol ratio as a prognostic marker in cardiovascular diseases. J Cell Physiol. 2018; 233(12): 9237-46.
[40]Enhos A, Cosansu K, Huyut MA, et al. Assessment of the Relationship between Monocyte to High-Density Lipoprotein Ratio and Myocardial Bridge. Arq Bras Cardiol. 2019; 112 (1): 12-17.

[41]Ekizler FA, Cay S. A novel marker of persistent left ventricular systolic dysfunction in patients with peripartum cardiomyopathy: monocyte count- to- HDL cholesterol ratio. BMC Cardiovasc Disord. 2019; 19 (1):114.

[42]Demirbas A, Kaya Islamoglu ZG. Can decreased monocyte to HDL-cholesterol ratio be a marker indicating the antiinflammatory effect of the colchicine in Behcet's disease? A preliminary study. Dermatol Ther. 2020; 33(6): e14013.

[43] Sirin MC, Korkmaz S, Erturan I, et al. Evaluation of monocyte to HDL cholesterol ratio and other inflammatory markers in patients with psoriasis. An Bras Dermatol. 2020; 95 (5):575-82.

[44] Katz JD, Agrawal S, Velasquez M. Getting to the heart of the matter: osteoarthritis takes its place as part of the metabolic syndrome. Curr Opin Rheumatol. 2010; 22 (5): 512-19.

[45] Gkretsi V, Simopoulou T, Tsezou A. Lipid metabolism and osteoarthritis: lessons from atherosclerosis. Prog Lipid Res. 2011; 50 (2):133-40.

[46] Schwager JL, Nevitt MC, Torner J, et al. Is there an association of serum low density lipoprotein, high density lipoprotein or total cholesterol with development of knee osteoarthritis? Arthritis Care Res (Hoboken). 2020:10.1002/acr.24455.

[47] Irshad K, Afzal MN. Comparison of serum lipid levels among patients suffering from osteoarthritis in Pakistan. Rawal Med J. 2014; 39(1): 6-9. 